\title{
Occurrence of Intestinal Pseudo-obstruction in a Brainstem Hemorrhage Patient
}

\author{
Sang-jee Lee, M.D., In-hun Na, M.D., Eun-seok Choi, M.D., Sung-hee Jung, M.D., Jong-soo Yoon, M.D.
}

Department of Rehabilitation Medicine, Daejeon St. Mary's Hospital, The Catholic University of Korea, Daejeon 301-723, Korea

Intestinal pseudo-obstruction is a massive colonic dilation with signs and symptoms of colonic obstruction, but without a mechanical cause. A 49-year-old female patient complained of nausea, vomiting, and abdominal distension 1 month after a massive brainstem hemorrhage. No improvement was seen with conservative treatments. An extended-length rectal tube was inserted to perform glycerin enema. In addition, bethanechol (35 mg per day) was administered to stimulate colonic motility. The patient's condition gradually improved over a 2-month period without any surgical intervention. Extended length rectal tube enema and bethanechol can be used to improve intestinal pseudo-obstruction in stroke patients.

Key Words Intestinal pseudo-obstruction, Rectal tube, Bethanechol

\section{INTRODUCTION}

Intestinal pseudo-obstruction is an intestinal obstruction symptom that occurs due only to movement disorder without intestinal mechanical stricture; the main cause in patients with brain lesions is known to be extrinsic autonomic nervous system abnormality. ${ }^{1}$ In stroke patients, air-fluid levels cannot be observed, and its vague physical signs and symptoms, intestinal pseudo-obstruction can be incorrectly diagnosed as a simple case of constipation and lead to a delay of correct diagnosis. Simple consti-

Received August 24, 2010; Accepted May 30, 2011

Corresponding author: In-hun Na

Department of Rehabilitation Medicine, Daejeon St. Mary's Hospital, The Catholic University of Korea, 520-2, Daeheung-dong, Jung-gu, Daejeon 301-723, Korea

Tel: +82-42-220-9650, Fax: +82-42-252-6807, E-mail: nih81@naver.com (c) This is an open-access article distributed under the terms of the Creative Commons Attribution Non-Commercial License (http:// creativecommons.org/licenses/by-nc/3.0) which permits unrestricted noncommercial use, distribution, and reproduction in any medium, provided the original work is properly cited.

Copyright () 2012 by Korean Academy of Rehabilitation Medicine pation is common and occurs in $55 \%$ of stroke patients during the initial 4-weeks after stroke, and can be caused by bed rest, medications, dehydration, and dysphagia. ${ }^{2}$ Unlike constipation, intestinal pseudo-obstruction is a serious ailment that can lead to complications such as megacolon, bowel perforation, peritonitis, and sepsis, ${ }^{3}$ and except for intravenous injection of neostigmine, the effectiveness of conservative treatments is limited. Here, we report a case where the condition of a patient with intestinal pseudo-obstruction due to extrinsic autonomic nervous system abnormality that was caused by brainstem hemorrhage showed improvement with the use of bethanechol without surgery, by inserting and leaving an extended-length rectal tube in the patient and repeatedly conducting decompressions and enemas.

\section{CASE REPORT}

A 49-year-old female patient visited the department of rehabilitation 1 month after suffering a cerebellar 
hemorrhage (Fig. 1) affecting more than two thirds of her anterior ventral of pons on both sides, midbrain, and medullar right of cerebellum. The upper and lower extremity strengths were MRC (medical research council) right 2 and left $0-1$, and there was ataxia on her right side. The patient was able to communicate by blinking her eyes, had limb and facial paralysis, dysarthria, dysphagia, sweating disorder, and had a Levin tube and the urinary catheter installed. She had a body temperature of $36.5^{\circ} \mathrm{C}$, pulse of 82 beats $/ \mathrm{min}$, and average blood pressure of 140/80 mmHg, which was not well controlled. Before visiting the hospital, the patient hadn't had bowel movement for more than 7 days and she complained of nausea, vomiting, and abdominal pain. She was experiencing abdominal distension, but abdominal tenderness and rebound tenderness were not observed, and had low bowel sounds. In a plain radiograph, the colon and small intestine were filled with gas and feces, and the ascending colon's diameter was increased to $95 \mathrm{~mm}$ (Fig. 2). On computed tomography, there were no tumors or vascular abnormalities that would cause mechanical obstruction in the intestinal tract, and there wasn't any endoscopic finding in the stomach. The blood tests showed hemoglobin $(11.8 \mathrm{~g} / \mathrm{dl})$, leukocytes $\left(9,500 / \mathrm{mm}^{3}\right)$, ESR (54), and CRP (0.88). In biochemical tests including an electrolytespecific test; there were no specific findings. A Clostridium difficile toxin test was negative. Although fasting, fluid therapy, bowel stimulant, intestinal massage, and

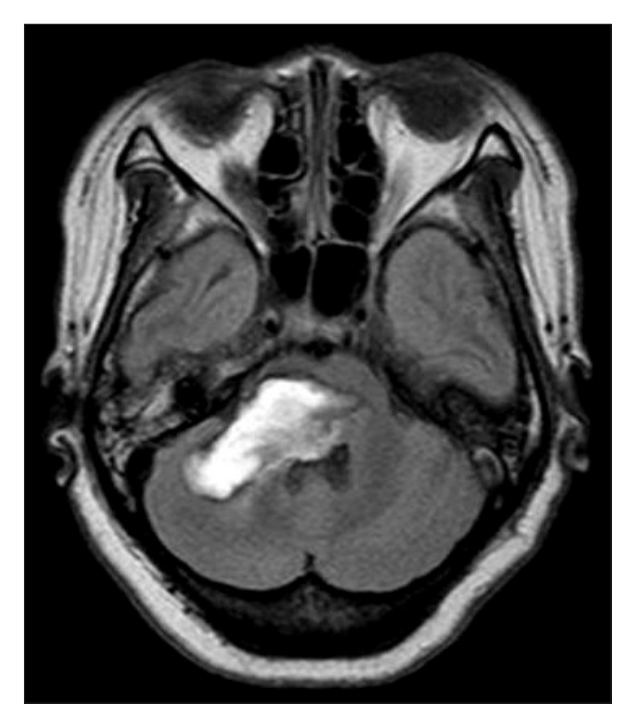

Fig. 1. Brain MRI of the patient (2 months after onset) shows massive brainstem hemorrhage involving area from midbrain, pons to cerebellum in axial T2 weighted FLAIR image. glycerin enema using an installed rectal tube were conducted, none of these procedures were effective. Starting on day 9 after admission, glycerin enema was conducted using a $170 \mathrm{~cm}$ extended-length rectal tube. The extended-length rectal tube was inserted with 4 French $45 \mathrm{~cm}$ rubber intestinal tubes overlapped $5 \mathrm{~cm}$ with one another and wound with Tegaderm ${ }^{\mathrm{TM}}$ (Healthcare, 3M). At 1-2 day intervals, enemas were repeatedly administered for 2 weeks. However, after 2 weeks, enemas were done less often and yet the oral dietary food intake was increased, hence, the symptoms worsened. Erythromycin (500 mg) was orally administered 4 times daily and the patient showed rapid improvement of symptoms, but due to the increased oral dietary food intake, nausea, vomiting, and gas distension were observed. To ease the condition, metoclopramide (10 mg) was intravenously injected 2 times daily and erythromycin was changed to bethanechol and administered. Bethanechol was administered at a starting dose of $5 \mathrm{mg}$, and then increased to 3 times daily or a total of more than $30 \mathrm{mg}$, along with a 1-2 times weekly enemas using the extended-length rectal tube, and regular stool movement was possible. After enemas, the extended-length rectal tube was forced out along with feces, and regular rectal tubes were then used. Common side effects of bethanechol such as sweating, flushing, and hypotension were not observed, and the dosage was reduced to $35 \mathrm{mg}$ and maintained after observing drooling of saliva at a dose of $40 \mathrm{mg}$. After 1 month of treat-

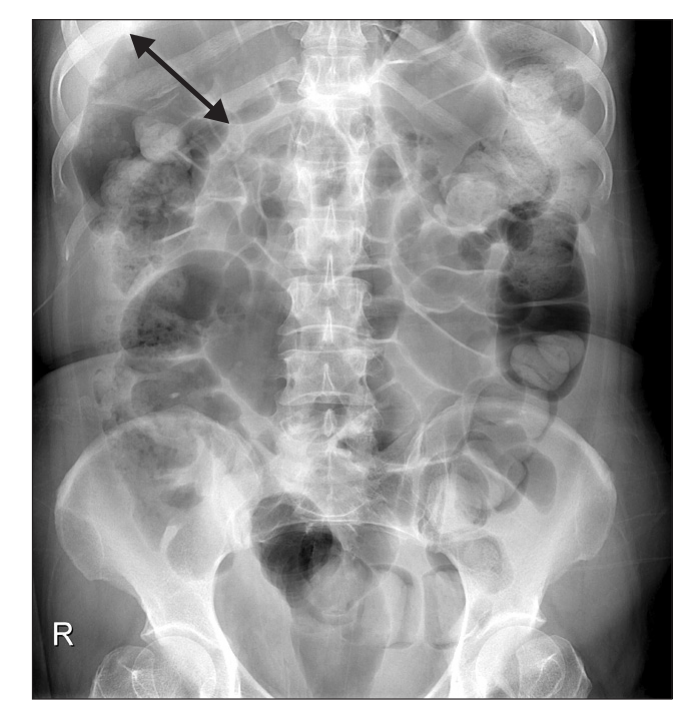

Fig. 2. Plain radiograph of a patient shows fecal impaction and marked gaseous dilatation in large and small bowel. Maximum diameter in ascending colon was $95 \mathrm{~mm}$. 
ments starting from the $75^{\text {th }}$ day after the first admission, the patient's symptoms did not worsen even without the use of rectal tubes. At 3 months after the first admission, stool movement was possible by using bisacodyl $(20 \mathrm{mg})$ once every 2-3 days. After 9 months, when the patient was re-admitted for rehabilitation treatment, her right-side muscle strength was grade 3 . And there was an improvement of dysphagia sufficient for her to swallow porridge. She was able to urinate by herself and there was frequent urination. When her amount of exercise was reduced, although bowel obstruction symptoms such as gas bloating were observed during abdominal radiography, they were easily improved after a daylong use of rectal tubes and treatment with magnesium hydroxide, mosapride, and bisacodyl suppositories.

\section{DISCUSSION}

Occurrences of intestinal pseudo-obstruction can be divided into 2 groups: causes of which are central and extrinsic autonomic nervous system anomalies of the central nervous system ${ }^{1,3}$ such as stroke, spinal cord injury, Parkinson's disease, multiple sclerosis, and Alzheimer's disease; and peripheral causes such as diabetes which invades the plexus. ${ }^{1}$ Based on analyses of 400 cases of intestinal pseudo-analysis, because the majority of cases were accompanied by complications such as infections including pneumonia and sepsis, surgery, metabolic diseases, cancer, respiratory ailment, renal failure, myocardial infarction, and heart failure, intestinal pseudo-obstruction could not infer information from admitted patients in the rehabilitation medicine department with these complications. ${ }^{4}$ The regions of the brain involved with control of the extrinsic autonomic nervous system are the prefrontal cortex, red nucleus of the stria terminalis, hypothalamic nucleus, nucleus of the amygdala, and medullar. ${ }^{5}$ In this study, the movement of the upper splenic flexure of the intestinal tract was reduced because of medullary lesions where the nucleus of the vagus nerve and nerves are located. The cause of intestinal pseudo-obstruction was a loss of parasympathetic nerve response or hyperactivity of parasympathetic nerve response due to an autonomic nervous system control anomaly. ${ }^{3}$ There is a previous report with findings of intestinal obstructions similar to those of this case caused by medullary lesions. ${ }^{6}$ The symptoms included abdominal pain, vomiting, bowel obstruction, diarrhea due to bacterial proliferation, and steatorrhea. ${ }^{3}$ In cases where bowel perforation occurs, the mortality rate reaches $40 \%,{ }^{4}$ but could not be distinguished by the symptoms only; tenderness was acute and accompanied by heat, or the white cell increase rate was higher, and special attention is required when the cecum diameter is more than $12 \mathrm{~cm}$ because this increases the possibility. ${ }^{3,7}$ Treatment is to adjust the electrolyte level anomaly by using a fluid therapy, and stop medications such as anticholinergic drugs, narcotics, and calcium channel blockers which reduce intestinal movement. For patients incapable of walking, it helps to have them grab their knees with their hands and change postures. ${ }^{7}$ Medicinal treatments for pseudo-obstruction include erythromycin, cisapride, neostigmine, bethanechol, octreotide, and domperidone. Although erythromycin, other than its antibiotic benefits, can affect the motilin receptor and promote intestinal tract movement, ${ }^{1,6,8}$ treatment effects on the distal are limited because the receptor exists proximal to the terminal ileal. ${ }^{8}$ Octreotide is a sustained-release somatostatin which promotes intestinal tract movement and blocks germ growth in the small intestine. ${ }^{1,8}$ Neostigmine has been proven to be effective in a randomized-blinded study. ${ }^{8}$ When conservative treatment has limitations, a slow intravenous injection of neostigmine ( $2 \mathrm{mg}$ ) is recommended, during which the monitoring of electrocardiogram, blood pressure, and hemodynamics are needed. ${ }^{3}$ We chose bethanechol, which can be administered orally, rather than a fast-acting intravenous injection medication for the patient with

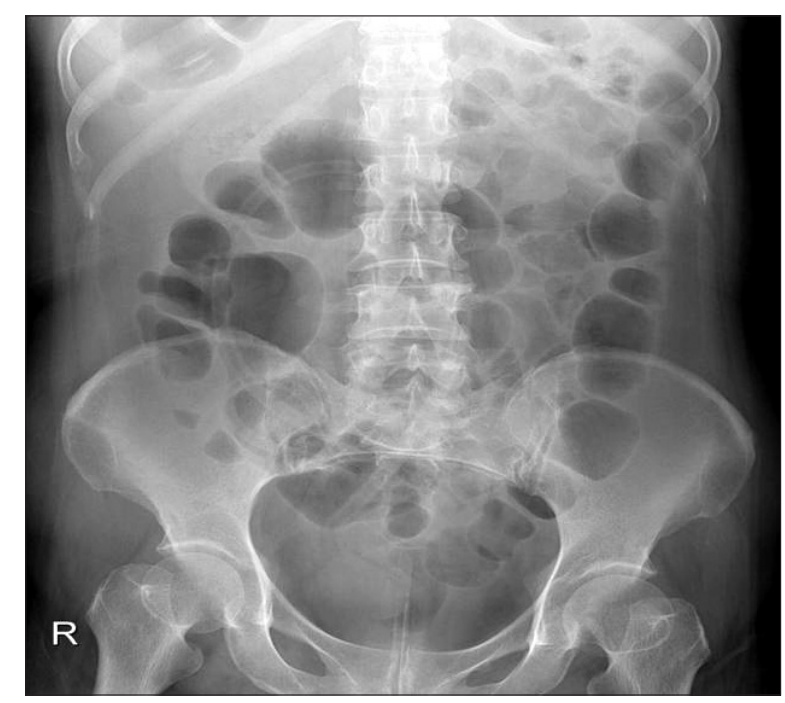

Fig. 3. Extended length rectal tube was inserted up to sigmoid colon with improvement of pseudo-obstruction. 
damaged control of the autonomous nervous system. Bethanechol is a drug that acts on the muscarinic receptor when it becomes hyperactive and acts on the bladder and abdominal organs. It is clinically similar to neostigmine, and is an agonist of the parasympathetic nerve, but induces partial contraction instead of promoting intestinal tract movement. ${ }^{8}$ Although there are reports of cases with subcutaneous injection of $10 \mathrm{mg}$, there are no written reports concerning oral administration of neostigmine. ${ }^{9}$ In the case report of this study, there was clinical improvement from continuing to administer regular enemas with bethanechol. When there was no improvement after administering medication, even though a pressure reduction procedure through colon endoscopy could be conducted, this was proven to be technically difficult, and a $1-5 \%$ occurrence rate of intestinal perforation complication was reported. ${ }^{7}$ In the case where an endoscope could not be utilized, there was report of improvement when a $140 \mathrm{~cm}$ extended-length rectal tube was used along with enema fluid. ${ }^{10}$ Although rectal tubes without the inducing power may become bent or experience difficulty going through the bends in the intestines, there is a low risk of sustaining perforated intestine and they don't require specialized skills. First, feces and intestinal gases in the lower intestinal tract are removed, and then the next insertion can penetrate slightly deeper into the upper part of intestine, which helps when conducting endoscopy. ${ }^{3}$ In this case study, the location of the rectal tube was verified to be near the top of the sigmoid colon bend (Fig. 3). Ostomy, a surgical procedure done under radiological guidance, is feasible; ${ }^{3}$ in a case where intestinal ischemia and intestinal perforation are of concern. The damaged part of the rectal tract can be removed and decompression procedures such as ostomy or cecostomy can be conducted. ${ }^{5}$ Although the underlying disease that reduced intestinal tract movement is not resolved by surgery, ostomy and cecostomy can improve nausea, vomiting and abdominal distension, and are effective means for delivering nutrition to the intestinal tract. However, the risk of recurrence and worsening of stroke must be understood by patients with acute stroke. This case study has discussed literature regarding intestinal pseudo-ob- struction. It is being reported because improvement was made by the rehabilitation department using relatively safe and easy methods.

\section{REFERENCES}

1. Antonucci A, Fronzoni L, Cogliandro L, Cogliandro RF, Caputo C, De Giorgio R, Pallotti F, Barbara G, Corinaldesi R, Stanghellini V. Chronic intestinal pseudoobstruction. World J Gastroenterol 2008; 14: 29532961

2. Su Y, Zhang X, Zeng J, Pei Z, Cheung RT, Zhou QP, Ling L, Yu J, Tan J, Zhang Z. New-onset constipation at acute stage after first stroke: incidence, risk factors, and impact on the stroke outcome. Stroke 2009; 40: 1304-1309

3. Saunders MD. Acute colonic pseudo-obstruction. Gastrointest Endosc Clin N Am 2007; 17: 341-360

4. Vanek VW, Al-Salti M. Acute pseudo-obstruction of the colon (Ogilvie's syndrome). An analysis of 400 cases. Dis Colon Rectum 1986; 29: 203-210

5. Wood JD, Alpers DH, Andrews PL. Fundamentals of neurogastroenterology. Gut 1999; 45 Suppl 2: II6-II16

6. Ito T, Sakakibara R, Sakakibara Y, Mori M, Hattori T. Medulla and gut. Intern Med 2004; 43: 1091

7. Fazel A, Verne GN. New solutions to an old problem: acute colonic pseudo-obstruction. J Clin Gastroenterol 2005; 39: 17-20

8. De Giorgio R, Barbara G, Stanghellini V, Tonini M, Vasina V, Cola B, Corinaldesi R, Biagi G, De Ponti F. Review article: the pharmacological treatment of acute colonic pseudo-obstruction. Aliment Pharmacol Ther 2001; 15: 1717-1727

9. Law NM, Bharucha AE, Undale AS, Zinsmeister AR. Cholinergic stimulation enhances colonic motor activity, transit, and sensation in humans. Am J Physiol Gastrointest Liver Physiol 2001; 281: G1228-1237

10. Durai R, Ng PC. 'Flatus tube enema' as an alternative to flexible sigmoidoscopy in reducing colonic volvulus and pseudo-obstruction: letter. Int J Colorectal Dis 2009; 24: 725-726 